\title{
A resolvent condition implying power boundedness
}

by

BÉLA NAGY (Budapest) and

JAROSLAV ZEMÁNEK (Warszawa)

Abstract. The Ritt and Kreiss resolvent conditions are related to the behaviour of the powers and their various means. In particular, it is shown that the Ritt condition implies the power boundedness. This improves the Nevanlinna characterization of the sublinear decay of the differences of the consecutive powers in the Esterle-KatznelsonTzafriri theorem, and actually characterizes the analytic Ritt condition by two geometric properties of the powers.

1. Introduction. Let $T$ be a bounded linear operator on a complex Banach space $X$, with spectrum $\sigma(T)$. In recent years, the property

$$
\left\|T^{n}-T^{n+1}\right\| \rightarrow 0 \quad \text { as } n \rightarrow \infty
$$

has been studied by a number of authors (see especially [Es], [KT], [N1], [N2]). The obvious necessary condition

$$
\sigma(T) \subset\{|\lambda|<1\} \cup\{1\}
$$

is not sufficient for (1) in general (even in the two-dimensional case).

Assume that (2) is satisfied. Observe that the differences $T^{n}-T^{n+1}$ are the Taylor coefficients of

$$
(\lambda-1)(T-\lambda I)^{-1}=-I+\sum_{n=1}^{\infty}\left(T^{n-1}-T^{n}\right) \lambda^{-n} \quad(|\lambda|>1) .
$$

It turns out that the boundedness of this analytic function, that is, the condition

$$
\left\|(T-\lambda I)^{-1}\right\| \leq \frac{\text { const }}{|\lambda-1|} \quad(|\lambda|>1)
$$

already implies (1) (see Proposition below). This condition (and the possibility of extending it to a larger sector) is relevant to the quantitative behaviour in (1) (see [N1, Theorem 4.5.4], [N2, Theorem 2.1]). It can be

1991 Mathematics Subject Classification: 47A10, 47A35. 
traced back to $[R]$, where it was shown that (2) and (4) imply

$$
\left\|T^{n}\right\|=o(n) \quad \text { as } n \rightarrow \infty,
$$

an important condition occurring in uniform ergodic theory [D], [LZ]. As pointed out by Olavi Nevanlinna in [Z1, p. 376], a close look at Ritt's proof $[R]$ reveals that more precise information can be derived from (4). Let

$$
M_{n}(T)=\frac{I+T+\ldots+T^{n-1}}{n} \quad(n=1,2, \ldots) .
$$

Proposition. Let $T$ satisfy (4). Then

(i) $\left\|T^{n}\right\|=O(\log n)$ as $n \rightarrow \infty$;

(ii) $\left\|M_{n}(T)\right\|=O(1)$ as $n \rightarrow \infty$;

(iii) $\left\|T^{n}-T^{n+1}\right\| \rightarrow 0$ as $n \rightarrow \infty$.

Proof. Note that (4) implies (2). Write each of the three operators in question as the Cauchy integral over a Jordan curve $\Gamma=\Gamma_{1} \cup \Gamma_{2} \cup \Gamma_{3}$ surrounding $\sigma(T)$, where $\Gamma_{1}$ is contained in the open unit disc (except for the endpoints), $\Gamma_{2}$ consists of two arcs on the unit circle, at distance $\geq 1 / n$ from 1 , and $\Gamma_{3}=\{|\lambda-1|=1 / n,|\lambda| \geq 1\}$. Choose $\Gamma_{2}$ of small length $\varepsilon>0$, and consider $n>1 / \varepsilon$. Standard estimations of each of the three integrals, over each of the three parts of $\Gamma$ separately, yield the results.

Now, (5) follows immediately from (i) or (iii). Concerning (i) and (iii), see also [EHP, Theorem 2] and [Tad]. Property (ii), in turn, implies the so-called uniform Abel boundedness [GHu, Theorem 2]. Also, a related result seems to be [KT, Theorem 2].

Moreover, by (iii) and the Fatou-Riesz theorem [V], series (3) converges at each point of the unit circle different from $1\left({ }^{1}\right)$. Consequently, by [PóS, III. 246], 1 is not a pole of (3).

It is interesting to compare (4) with the (weaker) Kreiss condition

$$
\left\|(T-\lambda I)^{-1}\right\| \leq \frac{\text { const }}{|\lambda|-1} \quad(|\lambda|>1)
$$

which implies the weaker conclusions

$$
\begin{aligned}
& \left\|T^{n}\right\|=O(n), \\
& \left\|M_{n}(T)\right\|=O(\log n) \text { and }\left\|M_{n}(T)^{2}\right\|=O(1)
\end{aligned}
$$

as $n \rightarrow \infty$ (see [N1, p. 32], [N2, p. 248], [Rö1, pp. 20, 52], [StW, pp. 344, $351]$ ). These consequences of (6) do not require (2). Does (2) add stronger conclusions? Is it possible that (2) together with (6) implies a variant of (1)?

$\left({ }^{1}\right)$ We shall see in Section 3 that the resolvent actually extends analytically through these points to a larger sector.
The Kreiss condition (6) can be characterized in terms of boundedness of certain functions of $T$ (see [Sh], [StW, Theorem 6.I]). As regards the (stronger) Ritt condition (4), see Theorem below.

2. Some examples. It may be instructive to look at a few examples. Consider the Volterra operator

$$
(V f)(t)=\int_{0}^{t} f(s) d s
$$

on a suitable space of functions on $(0,1)$. Let $A=(I+V)^{-1}$ and $B=I-V$. Recall that $A$ and $B$ are similar [A2, p. 15].

If $X=L_{2}(0,1)$, then $\left\|A^{n}\right\|=1$ for $n=1,2, \ldots$ (see [Ha, Problem 150]). So $A$ satisfies (6), but not (4) as can be seen from the resolvent [N1, p. 27].

On the other hand, if $X=L_{1}(0,1)$, then $\left\|B^{n}\right\|$ is of order $n^{1 / 4}$, but $\left\|M_{n}(B)\right\|$ is bounded (see [Hi, p. 247]). This example justifies [MZ, Théorème 2]. By (i), the Ritt condition cannot hold (which can also be seen directly from the resolvent as before). See also [A2], [Py].

An example satisfying (iii), but neither (i) nor (ii) $\left({ }^{2}\right)$, is [Św, Example 2].

To find an example that does satisfy (4) one can analyze [Rö2, Example 3.7], an example suggested by Béla Nagy for another purpose. Let $H$ be a separable Hilbert space with orthonormal bases $\left\{e_{k}\right\}$ and $\left\{f_{k}\right\}$. Let $X=H \oplus H$, and let $\left\{\alpha_{k}\right\}$ and $\left\{\beta_{k}\right\}$ be sequences in $(0,1)$ such that $\alpha_{k} / 1$ and $\beta_{k} \searrow 0$ as $k \rightarrow \infty$. For $e=\sum_{k} \xi_{k} e_{k}$ and $f=\sum_{k} \eta_{k} f_{k}$, the operator $T$ on $X$ is defined by

$$
T(e \oplus f)=\sum_{k}\left(\alpha_{k} \xi_{k}+\beta_{k} \eta_{k}\right) e_{k} \oplus f
$$

Then $\sigma(T)=\left\{\alpha_{k}\right\} \cup\{1\}$, and $\alpha_{k}$ are Fredholm points. Next,

$$
T^{n}\left(e_{k} \oplus f_{k}\right)=\left(\alpha_{k}^{n}+\frac{1-\alpha_{k}^{n}}{1-\alpha_{k}} \beta_{k}\right) e_{k} \oplus f_{k}
$$

and

$$
T^{n}(I-T)(e \oplus f)=\sum_{k} \alpha_{k}^{n}\left[\left(1-\alpha_{k}\right) \xi_{k}-\beta_{k} \eta_{k}\right] e_{k} \oplus 0 .
$$

Formula (3) now reads

(9) $(\lambda-1)(T-\lambda I)^{-1}(e \oplus f)=-(e \oplus f)+\sum_{k}\left(\frac{1-\alpha_{k}}{\lambda-\alpha_{k}} \xi_{k}-\frac{\beta_{k}}{\lambda-\alpha_{k}} \eta_{k}\right) e_{k} \oplus 0$,

$\left({ }^{2}\right)$ As observed by Urszula Skórnik. 
which can be verified by applying $T-\lambda I$ to both sides. Note that $\left|1-\alpha_{k}\right|<$ $\left|\lambda-\alpha_{k}\right|$ for $|\lambda|>1$. So if

$$
\sup _{k} \frac{\beta_{k}}{1-\alpha_{k}}<\infty
$$

then the right-hand side of (9) is bounded over $\{|\lambda|>1,\|e \oplus f\|=1\}$, and (4) holds. At the same time, however, (7) and (10) imply that $\sup _{n}\left\|T^{n}\right\|<\infty$ ! Passing to the Calkin algebra yields the identity. This example motivates an improvement of the above Proposition (see Section 3 below). Moreover, formulas (7) and (9) indicate a connection between the growth of the powers and the growth of the resolvent, depending on the growth of $\beta_{k} /\left(1-\alpha_{k}\right)$ as $k \rightarrow \infty$; it would be interesting to find a general form of this phenomenon. What is the characterization of (1) in this example?

A simple construction of an unbounded (not closable) operator satisfying (4) appears in [TaY, Example 3.7] though the resolvent operators are defined there only on a dense subspace. This indicates the strength of the requirement that the spectrum be small.

\section{The main result. For $\delta>0$ let}

$$
K_{\delta}:=\left\{\lambda=1+r e^{i \theta}: r>0,|\theta|<\frac{\pi}{2}+\delta\right\} .
$$

Curiously enough, it turns out that estimate (4) extends, with a larger constant, to some sector $K_{\delta}$. This is due to the particular form of the Taylor coefficients of the resolvent (the powers of a fixed operator). Since this observation is the key towards understanding and generalizing the example studied in Section 2, we include the simple proof below (see also [F, Lemma 4.2.3] or $[\mathrm{Pa}, \mathrm{p} .62])$.

\section{LEMMA. Suppose (4). Then}

$$
\left\|(T-\lambda I)^{-\mathbf{1}}\right\| \leq \frac{M}{|\lambda-1|} \quad\left(\lambda \in K_{\delta}\right)
$$

for some strictly positive constants $\delta$ and $M$. that

Proof. Let $A=T-I$. If we denote by $C$ the constant in (4), it follows

and

$$
\sigma(A) \subset\{\lambda:|\lambda+1|<1\} \cup\{0\}
$$

$$
\left\|(A-\lambda I)^{-1}\right\| \leq \frac{C}{|\lambda|} \text { for }|\lambda+1|>1
$$

In particular, this estimate holds for each $\lambda_{0}$ with $\operatorname{Re} \lambda_{0}=0$ and $\operatorname{Im} \lambda_{0} \neq 0$.
Since

$$
(A-\lambda I)^{-1}=\sum_{n=0}^{\infty}\left(A-\lambda_{0} I\right)^{-n-1}\left(\lambda-\lambda_{0}\right)^{n}
$$

whenever

$$
\left|\lambda-\lambda_{0}\right|\left\|\left(A-\lambda_{0} I\right)^{-1}\right\|<1,
$$

we see that $(A-\lambda I)^{-1}$ exists for all $\lambda$ with $\operatorname{Im} \lambda=\operatorname{Im} \lambda_{0}$ and $|\operatorname{Re} \lambda|<\left|\lambda_{0}\right| / C$. Since $\lambda_{0} \neq 0$ was arbitrary on the imaginary axis, it follows that $(A-\lambda I)^{-1}$ exists for all $\lambda \in K_{\varepsilon}-1$, where $\tan \varepsilon=1 / C$.

To obtain an appropriate estimate, fix a $\delta \in(0, \varepsilon)$ so that $\tan \delta=q / C$ for some $q \in(0,1)$. Consider a $\lambda \in K_{\delta}-1$ with $\operatorname{Re} \lambda<0$. Let $\lambda_{0}=i \operatorname{Im} \lambda$. Then

$$
\left|\lambda-\lambda_{0}\right|\left\|\left(A-\lambda_{0} I\right)^{-1}\right\|<q<1
$$

hence

$$
\left\|(A-\lambda I)^{-1}\right\| \leq\left\|\left(A-\lambda_{0} I\right)^{-1}\right\| \sum_{n=0}^{\infty} q^{n} \leq \frac{C}{\left|\lambda_{0}\right|(1-q)}<\frac{C}{|\lambda|(1-q) \cos \delta} .
$$

Thus, letting $M=C /((1-q) \cos \delta)=\sqrt{C^{2}+q^{2}} /(1-q) \geq C$ and going back to the operator $T$, we get the claim.

The above Lerama improves the second part of [N1, Theorem 4.5.4] to the effect that it actually suffices to assume the Ritt condition outside the unit disc only. Now, this result yields the power boundedness as well as the sublinear decay of the differences of the consecutive powers. Thus we get the following strengthening of parts (i) and (iii) in the above Proposition. (How could (ii) be improved?) The converse follows from [N2, Theorem 2.1] (see also [Go, Theorem I.5.4]).

THEOREM. If (4) holds, then

$$
\sup _{n}\left\|T^{n}\right\|<\infty
$$

where $n=1,2, \ldots$

Conversely, (12) and (13) together imply (4).

We note that (13) characterizes the essentially quickest possible convergence (1), in view of [N1, Theorem 4.5.1] and [Z1, p. 373]. In general, this convergence can be arbitrarily slow [ARa, Theorem 4.2]. Is it possible that (13) may imply (12) or at least (14) below? All the examples mentioned in Section 2 do suggest so. Moreover, (12) is actually not used in the proof of 
the first part of [N1, Theorem 4.5.4]. For Riesz operators, even (1) (via (5)) implies (12) by [Z1, Theorem 7].

Since $\left\|(T-\lambda I)^{-1}\right\| \geq 1 / \operatorname{dist}(\lambda, \sigma(T))$, we see that (4) corresponds to the slowest possible growth of the resolvent at $1 \in \sigma(T)$. This adds further interest to the question about the relation between (4) and (13), and motivates the more general problem of relating the rate of growth of the resolvent at 1 to the rate of convergence in (1).

In the following, (14) comes directly from (12) as in [N2, p. 251] or by [A1, Lemma 2], and (15) from (13) as in [N2, Theorem 2.1].

COROLLARY. If (4) holds, then

$$
\sup _{t \geq 0}\left\|e^{t(T-I)}\right\|<\infty
$$

and

$$
\left\|(T-I) e^{t(T-I)}\right\|=O\left(\frac{1-e^{-t}}{t}\right) \quad \text { for } t>0 .
$$

Is there a kind of converse also here? In particular, does (14) imply back (12) at least in the case where $\sigma(T)=\{1\}$ ? We note that (14) implies

$$
\left\|(T-\lambda I)^{-1}\right\| \leq \frac{\text { const }}{\operatorname{Re} \lambda-1} \quad(\operatorname{Re} \lambda>1)
$$

by estimating the Laplace integral formula for the resolvent [Pa, p. 25], while (12) implies (6) by estimating the Neumann series. The proof of (ii) $\Rightarrow$ (iv) in [N2, Theorem 2.1] shows that (14) and (15) together imply (11).

It would be interesting to know what is the optimal condition that has to be added to (4) in order to get the Gelfand conclusion $T=I$. Results of this kind and the corresponding references can be found in the survey [Z1]. See also [Z2].

4. Unbounded operators. The results of Section 3 complement the theory of analytic semigroups $[\mathrm{F}],[\mathrm{Go}],[\mathrm{Pa}]$ providing additional information about the generator. To show this, suppose that $T$ is a linear operator (not necessarily closed or densely defined) such that $\left({ }^{3}\right)$

$$
\left\|(T-\lambda I)^{-1}\right\| \leq \frac{\text { const }}{|\lambda|} \quad(|\lambda|>K) \text {. }
$$

Then $T$ must be bounded on $X$. (This improves the statements in [Go, p. 38] and $[\mathrm{Ki}, \mathrm{p} .60]$.)

$\left({ }^{3}\right)$ Here we understand that the inverse is a bounded operator on $X$, as in [Go, p. 13].
Indeed, since $(T-\lambda I)^{-1}$ is a closed operator for large $|\lambda|$, so is $T-\lambda I$, hence $T$ itself. It follows from (16) that the Laurent series of the resolvent around infinity has the form

$$
(T-\lambda I)^{-1}=\sum_{n=1}^{\infty} A_{n} \lambda^{-n}
$$

where the coefficients $A_{n}$ are bounded linear operators on $X$ satisfying

$$
T \sum_{n=1}^{\infty} A_{n} \lambda^{-n}=T(T-\lambda I)^{-1}=\lambda(T-\lambda I)^{-1}+I=\sum_{n=1}^{\infty} A_{n} \lambda^{-n+1}+I .
$$

For $\lambda \rightarrow \infty$ both series above converge, and the closedness of $T$ yields $-A_{1}=I$.

Thus, the Riesz-Taylor spectral projection corresponding to the bounded part of the extended spectrum of $T$ is $I$, hence the extended spectrum of $T$ is bounded. Consequently, $T$ is a bounded linear operator on $X$ by [Tay, Theorem 5.7-B].

In other words, the results of Section 3 apply to every linear operator satisfying (4).

Acknowledgements. In the recent paper [L], Yurii Lyubich also proves that (4) implies (12) and (13). Also Olavi Nevanlinna has claimed this answer to our original question, together with a converse. We are grateful to A. Bobrowski, K. M. Przyłuski and Yu. Tomilov for pointing out the connections with $[\mathrm{Ki}],[\mathrm{F}]$ and $[\mathrm{EHP}]$, respectively.

\section{References}

[A1] G. R. Allan, Sums of idempotents and a lemma of N. J. Kalton, Studia Math. 121 (1996), 185-192.

[A2] - Power-bounded elements and radical Banach algebras, in: Linear Operators, J. Janas, F. H. Szafraniec and J. Zemánek (eds.), Banach Center Publ. 38, Inst. Math., Polish Acad. Sci., 1997, 9-16.

[ARa] G. R. Allan and T. J. Ransford, Power-dominated elements in a Banach algebra, Studia Math. 94 (1989), 63-79.

[D] N. D unford, Spectral theory. I. Convergence to projections, Trans. Amer. Math. Soc. 54 (1943), 185-217.

[EHP] P. Erdös, F. Herzog and G. Piranian, On Taylor series of functions regular in Gaier regions, Arch. Math. (Basel) 5 (1954), 39-52.

[Es] J Esterle, Quasimultipliers, representations of $H^{\infty}$, and the closed ideal problem for commutative Banach algebras, in: Radical Banach Algebras and Automatic Continuity (Long Beach, Calif., 1981), J. M. Bachar, W. G. Bade, P. C. Curtis

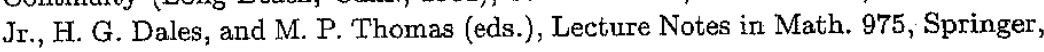
$1983,66-162$.

[F] H. O. Fattorini, The Cauchy Problem, Addison-Wesley, Reading, Mass., 1983. 
[Go] J. A. Goldstein, Semigroups of Linear Operators and Applications, Oxford Univ. Press, New York, 1985.

[GHu] J. J. Grobler and C. B. Huijsmans, Doubly Abel bounded operators with single spectrum, Quaestiones Math. 18 (1995), 397-406.

[Ha] P. R. Halmos, A Hïlbert Space Problem Book, Van Nostrand, Princeton, 1967.

[Hi] E. Hille, Remarks on ergodic theorems, Trans. Amer. Math. Soc. 57 (1945) $246-269$.

[KT] Y. Katznelson and L. Tzafriri, On power bounded operators, J. Funct. Anal. 68 (1986), 313-328.

[Ki] J. Kisyński, On resolvents and semigroups associated with the Dirichlet problem for an elliptic differential operator of second order with a Lévy perturbation Wydawnictwa Uczelniane Politechniki Lubelskiej, Politechnika Lubelska, Lublin, 1998.

[L] Yu. Lyubich, Spectral localization, power boundedness and invariant subspaces under Ritt's type condition, Studia Math. 134 (1999), 153-167.

[LZ] Yu. Lyubich and J. Zemánek, Precompactness in the uniform ergodic theory, ibid. 112 (1994), 89-97.

[MZ] M. Mbekhta et J. Zemánek, Sur le théorème ergodique uniforme et le spectre, C. R. Acad. Sci. Paris Sér. I Math. 317 (1993), 1155-1158.

[N1] O. Nevanlinna, Convergence of Iterations for Linear Equations, Birkhäuser Basel, 1993.

[N2] - , On the growth of the resolvent operators for power bounded operators, in: Linear Operators, J. Janas, F. H. Szafraniec and J. Zemánek (eds.), Banach Center Publ. 38, Inst. Math., Polish Acad. Sci., 1997, 247-264.

[Pa] A. Pazy, Semigroups of Linear Operators and Applications to Partial Differential Equations, Springer, New York, 1983.

[PóS] G. Pólya und G. Szegő, Aufgaben und Lehrsätze aus der Analysis, Springer, Berlin, 1964.

Py] T. Pytlik, Analytic semigroups in Banach algebras and a theorem of Hille, CoIloq. Math. 51 (1987), 287-294.

[R] R. K. Ritt, A condition that $\lim _{n \rightarrow \infty} n^{-1} T^{n}=0$, Proc. Amer. Math. Soc. 4 (1953), 898-899.

[Rö1] H. C. Rönnefarth, On properties of the powers of a bounded linear operator and their characterization by its spectrum and resolvent, Dissertation, Technische Universität Berlin, Berlin, 1996

[Rö2] -, On the differences of the consecutive powers of Banach algebra elements, in: Linear Operators, J. Janas, F. H. Szafraniec and J. Zemánek (eds.), Banach Center Publ. 38, Inst. Math., Polish Acad. Sci., 1997, 297-314.

[Sh] A.L.Shields, On Möbius bounded operators, Acta Sci, Math. (Szeged) 40 (1978), 371-374.

[StW] J. C. Strikwerda and B. A. Wade, A survey of the Kreiss matrix theorem for power bounded families of matrices and its extensions, in: Linear Operators, J. Janas, F. H. Szafraniec and J. Zemánek (eds.), Banach Center Publ. 38, Inst. Math., Polish Acad. Sci., 1997, 339-360.

[Św] A. Swięch, A note on the differences of the consecutive powers of operators, ibid., 381-383.

[Tad] E. Tadmor, The resolvent condition and uniform power-boundedness, Linear Algebra Appl. 80 (1986), 250-252.

[TaY] K. Tanahashi and S. Y amagami, Spectral inclusion relations for $T, T \mid Y$, and T/Y, Proc. Amer. Math. Soc. 116 (1992), 763-768.

[Tay] A. E. Taylor, Introduction to Functional Analysis, Wiley, New York, 1958.

[V] G. Valir on, Fonctions Analytíques, Presses Universitaires de France, Paris, 1954.

[Z1] J. Zemánek, On the Gelfand-Hille theorems, in: Functional Analysis and Operator Theory, J. Zemánek (ed.), Banach Center Publ. 30, Inst. Math., Polish Acad. Sci., 1994, 369-385.

[Z2] -, Problem, in: Banach Algebras '97, Proc. 13th Internat. Conf. on Banach Algebras (Blaubeuren, 1997), E. Albrecht and M. Mathieu (eds.), de Gruyter, Berlin, 1998, 560

Mathematical Institute

Technical University of Budapest

Egry József u. 2, H. ép., II. em.

H-1521 Budapest XI, Hungary

E-mail: bnagy@math.bme.hu

Fax: $(36-1) 4633172$
Institute of Mathematics Polish Academy of Sciences Śniadeckich 8, P.O. Box 137 00-950 Warszawa, Poland E-mail: zemanek@impan.gov.pi Fax: (48-22) 6293997 